\title{
Cyclotron emission from accretion plasma columns in magnetic cataclysmic variable stars
}

\author{
B. Kalomeni ${ }^{1,2}$, E. R. Pekünlü ${ }^{2}$, and K. Yakut ${ }^{2,3}$ \\ 1 Department of Physics, İzmir Institute of Technology, İzmir 35430, Turkey \\ e-mail: belinda@astronomy.sci.ege.edu.tr \\ 2 University of Ege, Faculty of Science, Department of Astronomy and Space Sciences, 35100 Bornova, İzmir, Turkey \\ 3 Katholieke Universiteit Leuven, Instituut voor Sterrenkunde Celestijnenlaan 200B, 3001 Leuven, Belgium
}

Received 3 August 2004 / Accepted 15 April 2005

\begin{abstract}
Pure cyclotron spectra of polars produced during their low accretion states are deduced. We used the working hypothesis that the cyclotron emission is produced by electrons spiraling down the dipole magnetic field lines and forming an accretion plasma column on top of the magnetic pole of a white dwarf. The velocity distribution function of electrons emitting cyclotron radiation is assumed to be a bi-Maxwellian. Since the radiating electrons in a million-Gauss magnetic field seek their respective magnetic mirrors, the perpendicular components of their velocity vectors are assumed to be greater than the parallel ones in the radiation region. This assumption implies that the cyclotron radiation is emitted more or less in the perpendicular direction (to the local magnetic field). Then we investigated the contribution of the ordinary and the extraordinary wave modes to the luminosity. The model predictions seem to be consistent with observations. We present the model cyclotron spectra of a randomly chosen polar, UZ For, as a case study.
\end{abstract}

Key words. magnetic fields - plasmas - stars: white dwarfs - stars: magnetic fields

\section{Introduction}

"Polars" (AM Her systems) as magnetic Cataclysmic Variables (mCVs) form a subclass of CVs. They are close binary systems in which a secondary star, which is usually - but not always - a red dwarf, donates mass to the primary, which is a white dwarf (WD). The magnetic field intensity of WDs is in the range of 10-230 MG. Accreting matter is captured by the magnetosphere of WD at a distance of about $10 \mathrm{WD}$ radii and guided by it to the magnetic poles of the primary (Schwope 1995). If the accreting matter is threaded at about $10 \mathrm{WD}$ radii and the field is dipolar, the accretion spots at the footpoints of the field lines have offsets from the poles on the order of $10^{\circ}-15^{\circ}$. An accretion column forms close to the magnetic poles of WDs.

A number of polars exhibit cyclotron line emission in their spectra (e.g. Reimers et al. 1999). During their low states, accretion streams do not form shocks above the magnetic pole regions. However, it is claimed "that the optical/IR observations alone cannot verify whether or not the shock is present, in spite of the claims of many papers. The strongest evidence for the absence of a hot unobscurred post-shock region in mCVs comes from thermal soft X-ray observations. A "Bi-Maxwellian" electron distribution can be produced in a shock heated plasma, provided the rate of cyclotron cooling is faster than the rate of collisional energyexchange". Nevertheless, we assume that the interactions both between the charged particles (electrons and protons) themselves and with the magnetic field are not disturbed by shocks. In this case, a great deal of cyclotron emission is assumed to originate near the top of the magnetic poles of a dipole field of WD (see, e.g., Rousseau et al. 1996; Schwope et al. 1990). Although this is a widely accepted scenario, Wu \& Wickramasinghe (1992) consider a multipole field consisting of a dipole and a quadrupole component. In our study, however, we assume that the magnetic field of the WD in the cyclotron emission region is dipolar.

We do, however, take the magnitude of the dipole magnetic field as constant along the emission region, the linear extent of which is on the order of $10^{7} \mathrm{~cm}$ (Chanmugam \& Langer 1991). The magnetic field intensity of polars was inferred from the cyclotron lines which were identified first in the spectra of VV Pup (Visvanathan \& Wickramasinghe 1979; Wickramasinghe \& Meggitt 1982). Broadened humps in the optical and/or infrared part of the spectra of polars are identified as the harmonics of the cyclotron radiation. While the fundamental frequency or the first harmonic usually falls into the infrared, the higher harmonics modulate the spectra in the visible region. If more than one harmonic is identified in the spectra, then one can infer the intensity of the magnetic field in the accretion column near the magnetic pole using Eq. (1) below:

$\omega_{\mathrm{ce}}=\frac{e B}{m_{\mathrm{e}} c}=2 \pi c\left(\frac{1}{\lambda_{n+1}}-\frac{1}{\lambda_{n}}\right) \quad n=1,2,3, \ldots$ 
where $e$ is the electronic charge, $B$ the magnetic field intensity, $m_{\mathrm{e}}$ the mass of an electron, $c$ the speed of light, $\lambda$ the wavelength of the harmonic, and $n$ an integer giving the harmonic number (Burwitz et al. 1997). The greater the number of harmonics in the spectra, the more precisely the magnetic field intensity is measured. After having inferred the magnetic field intensity we can move on to identify the $n$th harmonic for a magnetic field $B$, at low temperature and viewing angle, $\theta=90^{\circ}$, with the help of the formula (Ferrario et al. 1996),

$\lambda_{n}=\frac{10710}{n}\left(\frac{10^{8} \mathrm{G}}{B}\right)$.

The cyclotron radiation is emitted by the accreting electrons, whose energies are assumed to be in the $0.5-10 \mathrm{keV}$ range in low accretion rate polars (Schwope et al. 2002). In this region the electron number density is on the order of $10^{16} \mathrm{~cm}^{-3}\left(\mathrm{Chanmugam}^{-}\right.$ \& Langer 1991).

In Sect. 2 we propose a new model which has the potential of accounting for the observed spectra of some 27 AM Her systems. We are also well aware of the fact that previous models, i.e., "bombardment solution" (Woelk \& Beuermann 1992, 1993), "shock solution" (Lamb \& Masters 1979), and "blobby accretion solution" (Kuijpers \& Pringle 1982), all have enjoyed success. Schwope (1995) goes on to argue that all three may be in operation simultaneously in different parts of accretion region of any individual system.

The "bombardment solution" relies on the free fall of protons. In this model, material from a red dwarf is deposited in the magnetic polar region of a WD after having been captured by its magnetosphere. The word "capture" implies that the dynamics of the accreting plasma is determined by both the Lorentz force and the gravitational force of the WD. Closer to the magnetic polar region the former strongly opposes the latter. This ratio gets larger in favor of gravitational force only when the perpendicular velocity of the accreting plasma becomes smaller. Only when the velocity has a purely parallel component does the mirror force vanish and particles acquire free-fall velocity. At the point of capture in the magnetosphere, about ten white dwarf radii particles assume a wide range of pitch angles. It is hard to justify an assumption that all particles enter the magnetosphere with a zero pitch angle, but in such a case no cyclotron radiation could be emitted. However, one can argue that, similar to the "curvature radiation" received from radio pulsars due to the curved nature of the "open" field lines near the magnetic polar region, particles radiate cyclotron radiation. The angular distribution of cyclotron radiation emitted by the particles in their low Landau orbitals is confined mostly in a rather narrow cone along the instantaneous velocity. Even in this situation, the cyclotron radiation emitted by these "free-falling" particles will point towards the surface of WD and could hardly be detected by an outside observer.

Therefore, the bombardment solution, relying on the free-falling particles in a magnetic field of tens or hundreds of a million Gauss without feeling the magnetic mirror force seems to us a rather strong simplification. Besides, this model assumes a Maxwellian velocity distribution function that is also questionable in the presence of a million Gauss magnetic field. That is why, we believe, Schwope et al. (1990) has raised the need for a non-Maxwellian nature to the electron velocity distribution function.

Recently, Schwope et al. (2002) raise a question as to how the matter "manage to reach the extreme locations - that is, $B=$ 64/80 MG - in particular if accretion is still driven by normal Roche-lobe overflow or e.g. by a stellar wind or by chromospheric mass ejection". As this suspicion arisen because the magnetic mirror force is not taken into account in these models where matter "free-falls"?

Our model can reconcile this "bombardment solution" if we are allowed to refer to solar flares. In a solar flare, the single population of accelerated electrons produce both hard X-ray patches in chromosphere via bremsstrahlung and microwaves through gyrosynchrotron radiation; the former comes from electrons with smaller pitch angle and the latter from trapped, high pitch angle electrons within flaring loop(s). Especially decimetric type III radio bursts display so-called $J, U$, and $N$ morphology in their dynamic spectra, which means that a magnetic flux tube can harbour both mirroring and precipitating particles. Cyclotron emission reduces the component of electron velocity perpendicular to the magnetic field and may cause a very rapid pitch angle diffusion which enhances particle precipitation. Again, in the solar case, soft X-rays are produced by "chromospheric evaporation" following the hard X-ray patches formation. The soft X-ray problem of AM Her systems may be solved by a similar scenario. Kuijper \& Pringle (1982) have proposed that the fluctuating soft X-rays might be produced by instationary accretion of dense blobs or filaments of matter which penetrate the sub-photospheric layers and then heat the photosphere from below. They also suggest that soft X-rays might be produced by a stream of free-falling particles, which we read as "particles with smaller pitch angles". These particles heat the atmosphere and cause the formation of a hot corona which is cooled by cyclotron radiation.

\section{Cyclotron emission model}

The method used in calculating the absorption or emission of radiation from a source is outlined in Barrett \& Chanmugam (1985). In the case of mCVs where the large Faraday rotation applies (Meggitt \& Wickramasinghe 1982), a system of four coupled equations in Stokes parameters $I, Q, U$, and $V$ is formed (Pacholczyk 1970). If one reasons a priori that the large Faraday rotation is present, then the four coupled equations are reduced to two uncoupled equations in the intensity of the ordinary $(\mathrm{O})$ and the extraordinary (X) wave modes (Ramaty 1969). Using this method, Barrett \& Chanmugam (1985) derived the equation of radiative transfer (see also, Chanmugam \& Dulk 1981; Barrett \& Chanmugam 1984). These authors assumed that the velocity 
distribution function of radiating particles is Maxwellian. Then using the Kirchhoff's law they obtained the absorption coefficients for both the wave modes in terms of the blackbody intensity and the emissivity (Barrett \& Chanmugam 1985). The absorption coefficient for cyclotron radiation obtained with these methods depends on four independent variables: $\omega$, the radiation frequency; $k T$ the plasma temperature; $\theta$ the angle between the magnetic field and the wave vector; and $\Lambda=\omega_{\mathrm{p}}^{2} L / \Omega_{\mathrm{ce}} c$ the dimensionless constant, where, $\omega_{\mathrm{p}}$ is the plasma frequency, $L$ the thickness of the source, and $\Omega_{\mathrm{ce}}$ the cyclotron frequency of the radiating particles (Rousseau et al. 1996). $\Lambda$ is the measure of self-absorption in optically thick plasmas. Self-absorption in the optically thin plasmas, as we assume in the present investigation, may be neglected (Bekefi 1966). Following the method outlined in the work of Chanmugam \& Dulk (1981), Schwope et al. (1990) calculated the absorption coefficients of ordinary and extraordinary modes and the intensity escaping to infinity. Then they found the total flux emitted to the half sphere by UZ For. Rousseau et al. (1996) then applied the same method to model the cyclotron spectra of UZ For.

In the present investigation, we follow a different method to model the observed pure cyclotron spectra of polars. We extended the Green function construction (Jackson 1975) to a dispersive medium. The appropriate dispersion relation of the ordinary and extraordinary modes contains all the information about the propagation characteristics. Once the Green function is known one can find the radiation field and the Poynting vector, and then determine the instantaneous flux of energy. The main objective of this paper is to derive the Green function in a dispersive medium for ordinary and extraordinary wave modes, which would produce the pure cyclotron spectra of polars. We deduced our formulae in low mass accretion state, when cyclotron cooling becomes dominant and no shock can form (Woelk \& Beuermann 1996).

\subsection{The green function for a dispersive medium}

In a single particle approximation, $G$ represents a wave created by a point source at $\boldsymbol{r}^{\prime}$ which is pulsed at time $t^{\prime}$, and this wave propagates onwards from the source with a velocity $c$. The signal then is observed at some field point $\boldsymbol{r}$ at time $t$. For a single particle case, the Fourier transform of the Green function reads (Boyd \& Sanderson 1969):

$G(k, \omega)=\frac{1}{\pi} \frac{1}{k^{2}-\left(\omega^{2} / c^{2}\right)}$.

The Green function satisfies the wave equation for each value of $\omega$. In Eq. (3) $k=\omega / c$ is the wave number associated with the wave frequency $\omega$. We are motivated by the comment that "In this form, the restriction of no dispersion is unnecessary" by Jackson (1975). If we transfer the single particle approximation to the one for a dispersive medium with $n_{\mathrm{e}}$ radiating particles, the retarded Green function becomes,

$G\left(r, t ; r^{\prime}, t^{\prime}\right)=\frac{n_{\mathrm{e}}}{4 \pi} \int \mathrm{d}^{3} \boldsymbol{k} \int \mathrm{d} \omega \frac{\exp \left[\mathrm{i} \boldsymbol{k} \cdot\left(\boldsymbol{r}-\boldsymbol{r}^{\prime}\right)-\mathrm{i} \omega\left(t-t^{\prime}\right)\right]}{k^{2}-\left(\omega^{2} / c^{2}\right)}$.

Electron cyclotron emission implies a strong wave - particle interaction. Therefore, the " $k^{2}-\left(\omega^{2} / c^{2}\right)$ " term should be determined by the dispersion relation for a specific wave mode. For this purpose, we adopt the assumption, i.e., $|\omega|^{2} \approx \Omega_{\mathrm{ce}}^{2} \approx c^{2} k^{2} \gg \omega_{\mathrm{pe}}^{2}$, which is readily shown to be justifiable (see also, Rousseau 1996), while the wave vector is nearly perpendicular to the local magnetic field and uses the dispersion relations for the extraordinary mode (Wu 1985),

$1-\frac{c^{2} k_{\perp}^{2}}{\omega^{2}}+\frac{\omega_{\mathrm{pe}}^{2}}{\omega^{2}} \int \mathrm{d}^{3} v\left(\Omega_{\mathrm{ce}} \frac{\partial F_{\mathrm{e}}}{\partial v_{\perp}}+k_{\|} v_{\perp} \frac{\partial F_{\mathrm{e}}}{\partial v_{\|}}\right) \frac{v_{\perp}\left(J_{1}^{\prime}\right)^{2}(b)}{\left(\omega-\Omega_{\mathrm{ce}} / \gamma-k_{\|} v_{\|}\right)}=0$

and for the ordinary mode (ibid),

$1-\frac{c^{2} k^{2}}{\omega^{2}}+\frac{\omega_{\mathrm{pe}}^{2}}{\omega^{2}} \int \mathrm{d}^{3} v\left(\Omega_{\mathrm{ce}} \frac{\partial F_{\mathrm{e}}}{\partial v_{\perp}}+k_{\|} v_{\perp} \frac{\partial F_{\mathrm{e}}}{\partial v_{\|}}\right) \frac{v_{\|}^{2} J_{1}^{2}(b)}{v_{\perp}\left(\omega-\Omega_{\mathrm{ce}} / \gamma-k_{\|} v_{\|}\right)}=0$,

where $J_{1}^{\prime}(b)=\mathrm{d} J_{1}(b) / \mathrm{d} b$ is the derivative of Bessel function; $b=k_{\perp} v_{\perp} / \Omega_{\mathrm{ce}} ; \gamma=\left(1-v^{2} / c^{2}\right)^{-1 / 2} ; v_{\|}$and $v_{\perp}$ are parallel and perpendicular (to the local magnetic field) velocity components, respectively; $\Omega_{\mathrm{ce}}=|e| B_{0} / m_{\mathrm{e}} c$ is the electron cyclotron frequency; $B_{0}$ is the magnetic field intensity; and $F_{\mathrm{e}}$ is the unperturbed electron velocity distribution function. In Eq. (4) we replace the " $k^{2}-\left(\omega^{2} / c^{2}\right)$ " term with its equivalent which will be derived from Eqs. (5) and (6).

Another motivation for our present investigation comes from the Schwope et al. (1990) paper wherein they pointed out the possible non-Maxwellian nature of the electron velocity distribution function. We follow this suggestion and assume that the unperturbed electron distribution function $F_{\mathrm{e}}$ is a bi-Maxwellian (Schmidt 1979):

$F_{\mathrm{e}}=n_{\mathrm{e}} \alpha_{\perp}^{2} \alpha_{\|} \pi^{-3 / 2} \exp \left[-\left(\alpha_{\perp}^{2} v_{\perp}^{2}+\alpha_{\|}^{2} v_{\|}^{2}\right)\right]$

where, $\alpha_{\|}=\left(2 k_{\mathrm{B}} T_{\mathrm{e} \|} / m_{\mathrm{e}}\right)^{-1 / 2}$ and $\alpha_{\perp}=\left(2 k_{\mathrm{B}} T_{\mathrm{e} \perp} / m_{\mathrm{e}}\right)^{-1 / 2}$ are the inverse of most probable speeds in parallel and perpendicular directions, respectively; $k_{\mathrm{B}}$ is the Boltzmann constant; $T_{\mathrm{e} \|}$ and $T_{\mathrm{e} \perp}$ are the parallel and perpendicular temperatures of electrons, respectively. 
The derivatives of $F_{\mathrm{e}}$ with respect to the parallel and perpendicular velocity components that appear in Eqs. (5) and (6) will be obtained from Eq. (7). In what follows, we drop the arguments of the Green function for the sake of brevity, but wherever it appears the arguments are implied. Now, let us write the Green function for the extraordinary mode:

$G=\frac{n_{\mathrm{e}}}{4 \pi^{3}} \int \mathrm{d}^{3} k \int \mathrm{d} \omega \frac{c^{2}}{\omega_{\mathrm{pe}}^{2}} \frac{\exp \left[\mathrm{i} \boldsymbol{k} \cdot\left(\boldsymbol{r}-\boldsymbol{r}^{\prime}\right)-i \omega\left(t-t^{\prime}\right)\right]}{\int \mathrm{d}^{3} v\left[-2 \alpha_{\perp}^{2} \Omega_{\mathrm{ce}} n_{\mathrm{e}} \alpha_{\|} \pi^{-3 / 2} v_{\perp} \exp \left(-\alpha_{\perp}^{2} v_{\perp}^{2}-\alpha_{\|}^{2} v_{\|}^{2}\right)\right]\left(v_{\perp} J_{1}^{\prime 2}(b) /\left(\omega-\Omega_{\mathrm{ce}} / \gamma-k_{\|} v_{\|}\right)\right)}$.

From the recurrence formulae of Bessel functions, we write

$J_{1}^{\prime 2}\left(\frac{k_{\perp} v_{\perp}}{\Omega_{\mathrm{ce}}}\right)=\frac{1}{4}\left(1-\frac{3}{4} \frac{k_{\perp}^{2} v_{\perp}^{2}}{\Omega_{\mathrm{ce}}^{2}}\right)$.

Since we consider a reduced velocity function (Vocks $\&$ Marsch 2002), we can make the relevant replacement, i.e., $\int \mathrm{d}^{3} v=$ $\int \mathrm{d} v_{\|} \int v_{\perp} \mathrm{d} v_{\perp}$. Then, using the recurrence formulae of commonly occurring integral, $h(n)=\int_{0}^{\infty} x^{n} \exp \left(-a x^{n}\right) \mathrm{d} x$, the first term of the $v_{\perp}$ integral,

$\int_{0}^{\infty} v_{\perp}^{3} \exp \left(-\alpha_{\perp}^{2} v_{\perp}^{2}\right) \mathrm{d} v_{\perp}=\left(2 \alpha_{\perp}^{4}\right)^{-1}$

and similarly, the second one,

$-\int_{0}^{\infty} \frac{3}{4} \frac{k_{\perp}^{2}}{\Omega_{\mathrm{ce}}^{2}} v_{\perp}^{5} \exp \left(-\alpha_{\perp}^{2} v_{\perp}^{2}\right) \mathrm{d} v_{\perp}=-\frac{3}{4} \frac{k_{\perp}^{2}}{\Omega_{\mathrm{ce}}^{2} \alpha_{\perp}^{6}}$

are obtained. The $v_{\|}$integral is calculated as,

$\int_{-\infty}^{+\infty} \frac{\exp \left(-\alpha_{\|}^{2} v_{\|}^{2}\right)}{\omega-\Omega_{\mathrm{ce}} / \gamma-k_{\|} v_{\|}} \mathrm{d} v_{\|}=-\frac{\sqrt{\pi}}{k_{\|}}\left(\frac{-k_{\|} \gamma}{\gamma \alpha_{\|} \omega-\Omega_{\mathrm{ce}} \alpha_{\|}}\right)$

If we make these substitutions in Eq. (8), we get the Green function for extraordinary mode as:

$G=\frac{c^{2}}{\pi^{2} \omega_{\mathrm{pe}}^{2} \Omega_{\mathrm{ce}}} \int \mathrm{d}^{3} k \int \mathrm{d} \omega \exp \left[\mathrm{i} \boldsymbol{k} \cdot\left(\boldsymbol{r}-\boldsymbol{r}^{\prime}\right)-\mathrm{i} \omega\left(t-t^{\prime}\right)\right] \frac{\left(\gamma \omega-\Omega_{\mathrm{ce}}\right)}{-1+(3 / 2)\left(k_{\perp} / \Omega_{\mathrm{ce}} \alpha_{\perp}\right)^{2}}$.

Let us put $\delta t=t-t^{\prime}$ and evaluate the $\omega$ integral as:

$\int \exp (-\mathrm{i} \omega \delta t)\left(\omega \delta t-\Omega_{\mathrm{ce}}\right) \mathrm{d} \omega=\gamma \frac{\exp (-\mathrm{i} \omega \delta t)}{\mathrm{i} \delta t}\left(-\omega-\frac{1}{\mathrm{i} \delta t}+\frac{\Omega}{\gamma}\right)$.

Now, let us evaluate the $k$ integral,

$\int \frac{\mathrm{d}^{3} k}{-1+(3 / 2)\left(k_{\perp} / \Omega_{\mathrm{ce}} \alpha_{\perp}\right)^{2}}$.

Since the orientation of transform space with respect to the coordinate space is arbitrary, we are allowed to let $\boldsymbol{R}=\boldsymbol{r}-\boldsymbol{r}^{\prime}$ be coincident with $k_{\|}$. In this case in $(k, \theta, \phi)$ coordinates, $k_{\perp x}=k \sin \theta \cos \phi ; k_{\perp y}=k \sin \theta \sin \phi ; k_{\|}=k \cos \theta$ and $\mathrm{d} k=k^{2} \sin \theta \mathrm{d} k \mathrm{~d} \theta \mathrm{d} \phi$. These relations allow us to write the $k$ integral as:

$$
\int \frac{\mathrm{d}^{3} k}{-1+\frac{3}{2}\left(k_{\perp} / \Omega_{\mathrm{ce}} \alpha_{\perp}\right)^{2}}=\int_{0}^{\infty} \mathrm{d} k \int_{60^{\circ}}^{80^{\circ}} \mathrm{d} \theta \int_{0}^{2 \pi} k^{2} \sin \theta \exp (\mathrm{i} \boldsymbol{k} \cdot \boldsymbol{R}) \frac{1}{-1+\frac{3}{2}\left(k_{\perp} / \Omega_{\mathrm{ce}} \alpha_{\perp}\right)^{2}} \mathrm{~d} \phi .
$$

As it is stated above that cyclotron radiation is emitted more or less perpendicularly to the local magnetic field lines, $\theta$ is the angle between $\boldsymbol{k}$ and $\boldsymbol{B}$. If we perform the $\theta$ and $\phi$ integrals, Eq. (16) becomes,

$$
\int \frac{\mathrm{d}^{3} k}{-1+\frac{3}{2}\left(k_{\perp} / \Omega_{\mathrm{ce}} \alpha_{\perp}\right)^{2}}=\left(\frac{2 \pi}{\mathrm{i} R}\right) \int_{0}^{\infty} \frac{\left(\mathrm{e}^{-0.5 \mathrm{i} k R}-\mathrm{e}^{0.175 \mathrm{i} k R}\right) k}{\left[3 /\left(2 \Omega_{\mathrm{ce}}^{2} \alpha_{\perp}^{2}\right)\right]\left(k_{\perp}^{2}-2 \Omega_{\mathrm{ce}}^{2} \alpha_{\perp}^{2} / 3\right)} \mathrm{d} k .
$$


For $\mp \sqrt{2 / 3} \Omega_{\mathrm{ce}} \alpha_{\perp}$ values of $k_{\perp}$, the denominator on the right hand side of Eq. (17) becomes zero. Therefore we should evaluate the residual contributions to the integral. With these contributions, the value of the $k$ integral becomes

$\int \frac{\mathrm{d}^{3} k}{-1+\frac{3}{2}\left(k_{\perp} / \Omega_{\mathrm{ce}} \alpha_{\perp}\right)^{2}}=\frac{8 \pi^{2} \Omega_{\mathrm{ce}}^{2} \alpha_{\perp}^{2}}{3 R}[\cos (0.5 R x)-\cos (0.175 R x)]$,

where $x=\mp \sqrt{2 / 3} \Omega_{\mathrm{ce}} \alpha_{\perp}$ is substituted for brevity. Here, we assume that the observer only receives the emission coming in a narrow angle range, i.e. $60^{\circ}-80^{\circ}$. For instance, in UZ For's spectrum the luminosity for $\theta=20^{\circ}$ and for $\theta=90^{\circ}$ differs by a factor of 7-8 for the 5 th harmonic. We discuss the angular dependence of the cyclotron radiation luminosity in Sect. 2.3 in detail. If we now substitute the values of Eqs. (14) and (18) into Eq. (13) we finally get the Green function for the extraordinary mode:

$G=-\frac{8 \Omega_{\mathrm{ce}} \alpha_{\perp}^{2} c^{2}}{3 R \omega_{\mathrm{pe}}^{2} \delta t}\left[\left(-\omega+\frac{\Omega_{\mathrm{ce}}}{\gamma}\right) \mathrm{i} \cos (\omega \delta t)-\frac{\sin (\omega \delta t)}{\mathrm{i} \delta t}\right][\cos (0.5 R x)-\cos (0.175 R x)]$.

Equation (19) is the general solution for the Green function. Once it is known, the electric component of the radiation field can be evaluated from the formula (Boyd \& Sanderson 1969):

$\boldsymbol{E}(\boldsymbol{r}, t)=-e \boldsymbol{n} \frac{\partial}{\partial R} \int G \mathrm{~d} t^{\prime}-\frac{e}{c^{2}} \frac{\partial}{\partial t} \int \boldsymbol{v}\left(t^{\prime}\right) G \mathrm{~d} t^{\prime}$

where $\boldsymbol{n}=\boldsymbol{R} / R$ is the unit vector directed from the position of the particle to the observer. Since the observation point is far away from the radiating electrons so that during a small acceleration interval, the changes in $\boldsymbol{n}$ and $\boldsymbol{R}$ may be assumed to be negligible. After some rather tedious but straightforward calculations we find the electric component of the radiation field as:

$\boldsymbol{E}=\frac{8 \Omega_{\mathrm{ce}} \alpha_{\perp}^{2} e c}{3 R \omega_{\mathrm{pe}}^{2} \delta t}\left[\left(-\omega+\frac{\Omega_{\mathrm{ce}}}{\gamma}\right) \mathrm{i} \cos (\omega \delta t)-\frac{\sin (\omega \delta t)}{\mathrm{i} \delta t}\right][\cos (0.5 R x)-\cos (0.175 R x)] \dot{\beta}$

where $\dot{\beta}$ is found from the Lorentz force as $\dot{\beta}=q v B / m c$. Since we assume a constant magnetic field and mono-energetic particles, it is explicit that $\dot{\beta}$ is constant. The right hand side of Eq. (21) has the dimension of $M^{1 / 2} / L^{1 / 2} T$ in Gaussian units, which is the dimension of electric field. As is well known, the electric field of a radiating particle is directly proportional to $\dot{\beta}$ and inversely proportional to $R$. The energy flux is given by the Poynting vector, $\boldsymbol{S}=(c / 4 \pi)|\boldsymbol{E}|^{2} \boldsymbol{n}$. The energy emitted to the unit solid angle is $\mathrm{d} P(t) / \mathrm{d} \Omega=(c / 4 \pi)|R \boldsymbol{E}(t)|^{2}=|\boldsymbol{A}(t)|^{2}$ where $\Omega$ is the solid angle, which should not be confused with the electron cyclotron frequency, $\Omega_{\mathrm{ce}}$.

It is also our concern to determine how the radiated energy is distributed in frequency. For this, one introduces the Fourier transform of $|\boldsymbol{A}(t)|^{2}$ and then using Parseval's theorem, the energy radiated per unit solid angle per unit frequency interval is obtained:

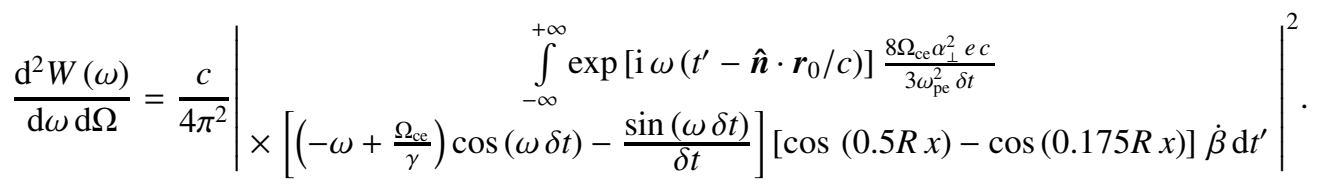

Now we suppose that the axes are so oriented that the observer is in the Oxz plane. Then the unit vector of the direction from the source to the observer is $\boldsymbol{n}=\sin \theta \boldsymbol{i}+\cos \theta \boldsymbol{k}$ where $\theta$ is the angle between the line of sight (los) and the magnetic field. The exponential term under the integral sign in Eq. (22) is given by Bekefi (1966) as

$\exp (\mathrm{i} x \sin y)=\sum_{l=-\infty}^{\infty} J_{l}(x) \exp (\mathrm{i} l y)$

where $J$ is the Bessel function. If we rearrange the exponential function under the integral sign in accordance with the Eq. (23), we get (Boyd \& Sanderson 1969),

$\exp \left\{\mathrm{i} \omega\left[t-\frac{\hat{\boldsymbol{n}} \cdot \boldsymbol{r}_{0}\left(t^{\prime}\right)}{c}\right]\right\}=\sum_{l=-\infty}^{\infty} J_{l}\left(\frac{\omega}{\Omega_{\mathrm{ce}}} \beta_{\perp} \sin \theta\right) \exp \left[\mathrm{i}\left(\omega-l \Omega_{\mathrm{ce}}-\omega \beta_{\|} \cos \theta\right) t\right]$.

Substitution of Eqs. (24) into (22) gives the energy radiated per unit solid angle per unit frequency interval in its final form:

$\frac{\mathrm{d}^{2} W(\omega)}{\mathrm{d} \omega \mathrm{d} \Omega}=\frac{4 B_{0} e m^{2} c^{3} T_{\mathrm{r}}}{9 \pi k_{\mathrm{B}}^{2} n_{\mathrm{e}} \Lambda T_{\perp}^{2} L}\left|\sum_{l=1}^{10} \times \underset{\substack{J_{l}\left(\frac{\omega}{\Omega_{\mathrm{ce}}} \beta_{\perp} \sin \theta\right) \\[\cos (0.5 R x)-\cos (0.175 R x)] \dot{\beta}}}{\left[\left(-\omega+\frac{\Omega_{\mathrm{ce}}}{\gamma}\right) \cos (\omega \delta t)-\frac{\sin (\omega \delta t)}{\delta t}\right]}\right|^{2} \delta\left(l \Omega_{\mathrm{ce}}-\omega\left[1-\beta_{/ / \cos \theta]),}\right.\right.$ 
where $T_{\mathrm{r}}$ is the radiation time in the observer's device (see, e.g. Boyd \& Sanderson 1969); and for ordinary mode we find;

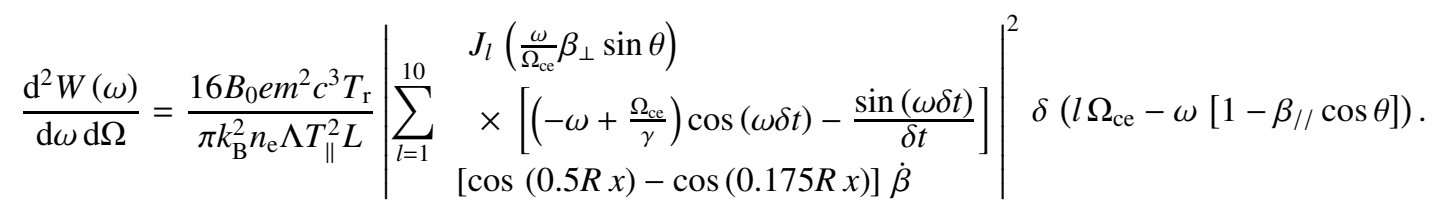

The first striking result of our analysis is that, while the luminosity of the extraordinary mode is inversely proportional to $T_{\perp}^{2}$, luminosity of the ordinary mode is inversely proportional to $T_{\|}^{2}$. Anisotropy manifests itself in the relevant mode's exclusive dependence on the perpendicular or parallel temperature. We further discuss this point in Sect. 2.3. Finally, by a suitable change of free parameters $N_{\mathrm{e}}, \Lambda, T_{\|}, T_{\perp}, L$, one can fit the model spectra to the observed one. We consider the summation of the first 10 terms in Eqs. (25) and (26), because inclusion of extra terms does not change the result too much. Equations (25) and (26) give the frequency spectrum of cyclotron emission in $\mathrm{O}$ and $\mathrm{X}$ modes. The spectrum consists of emission lines at frequencies, $\Omega_{\mathrm{ce}}, 2 \Omega_{\mathrm{ce}}, 3 \Omega_{\mathrm{ce}}$, and so on. Observations of the composite spectra of various polars show broad humps, which are identified as cyclotron emission lines. These lines are not discrete but instead broadened. Line widths sometimes are as broad as $1000 \AA$ (see e.g., Schwope et al. 1990).

When plotted in the frequency domain, the energy distribution shows broadened lines, the central frequencies of which are, as expected, equidistant. In the case study to be given in Sect. 2.3, however, we shall give the energy distribution in wavelength domain to make it easier to compare the results with observations.

We should say a few words about the time difference, $\delta t=t-t^{\prime}$. We interpret it as the ratio, $L / c$, where $L$ is the thickness of the slab containing the radiating electrons. Since after having been generated in the slab by electrons, energy is neither regenerated nor destroyed on its way to the observer then it is justifiable to place the observer just outside the slab.

If we first measure, by Zeeman effect, say, the magnetic field intensity of the WD companion of a polar (e.g., Bailey et al. 1985; Schmidt et al. 1983), then use the parameter ranges in AM Her binaries (i.e., $10^{14} \leq n_{\mathrm{e}} \leq 10^{16} \mathrm{~cm}^{-3}, 5 \mathrm{keV} \leq k T \leq 10 \mathrm{keV}$, and $60^{\circ} \leq \theta \leq 80^{\circ}$ and $\alpha_{\|} / \alpha_{\perp}=2.236$ ), and finally substitute these values into Eqs. (25) and (26), we get a spectrum consisting of broadened cyclotron lines. Then using Eqs. (1) and (2), we find the magnetic field intensity in the accretion column close to the surface of the WD. The inferred value of the field intensity can be compared with the value measured during a low state of accretion by Zeeman features (Wickramasinghe \& Ferrario 1995). In this study, we consider neither the variation of cyclotron spectra with respect to orbital phases nor the mass accretion rate yet, but will do so in a future study. As already been pointed out by Schwope et al. (1990), none of these parameters can be determined independently from the others: "a change in the spectra by variation of one parameter can, to some extent, be compensated by a suitable variation of the remaining ones".

\subsection{Case study - UZ For}

UZ For (EXO 033319-2554.2) is an eclipsing AM Herculis binary. It was discovered as an X-ray source during systematic analysis of EXOSAT soft X-ray data and subsequently identified as an AM Her binary by its X-ray and optical properties (Giommi et al. 1987; Beuermann et al. 1987; Bailey et al. 1987; Osborne et al. 1988). In low accretion state during the bright phase (0.70-0.11) when the main accretion region is in view, the optical continuum is modulated by intense cyclotron line emission (Schwope et al. 1990). A set of low-dispersion spectrophotometric observations covering a wavelength range of 3800-10000 $\AA$ was obtained on October 21, 1987 with the ESO/MPI $2.2 \mathrm{~m}$ telescope at La Silla. Clearly resolvable cyclotron harmonics were detected at phase interval 0.70-0.11. In Fig. 2 of Schwope et al. (1990) 3rd, 4th, and 5th harmonics are clearly seen.

Earlier, Wickramasinghe \& Megitt (1982) pointed out that in general the lower harmonics have higher opacity and become optically thick first. Later, Schwope et al. (1990) observed in UZ For spectra that the flux emitted in the 5th harmonic is greater than that of the 4th. They interpreted this feature as suggesting that harmonics with harmonic number $m \leq 4$ become optically thick, while those with $m \geq 5$ are optically thin. Our model spectra (see Fig. 1) confirm the existence of this general tendency quite clearly.

The authors describe the flux distribution by assuming a homogeneous plasma with $k T_{\text {cyc }} \cong 5-10 \mathrm{keV}$ in a field of $53 \mathrm{MG}$. Location of this region is reported as below the orbital plane at stellar colatitude $\delta_{1} \cong 155^{\circ}$. The possibility of the second emission region on the opposite hemisphere is not ruled out by Schwope et al. (1990). A faint phase emission line at $\sim 4740 \AA$ is identified as a cyclotron harmonic emitted by a plasma with $k T_{\text {cyc }} \cong 1 \mathrm{keV}$ from a field region with the magnetic field intensity of $75 \mathrm{MG}$ and a stellar colatitude $\delta_{2} \cong 10^{\circ}$. The offset in longitude between these two emission spots is reported as $\sim 80^{\circ}$. This means that at a certain phase interval both the magnetic poles are in view. In order to produce the observed spectra, we take the intensity of the pole as $53 \mathrm{MG}$. In that case, the thicknesses of slabs should be $1.3 \times 10^{6} \mathrm{~cm}$. The calculated central wavelengths of the harmonics are $\lambda_{3}=6780 \AA, \lambda_{4}=5090 \AA, \lambda_{5}=4070 \AA$, where the subscripts denote harmonic number. The observed locations of the central wavelengths are given by Schwope et al. (1990) and Rousseau et al. (1996) as $\lambda_{3}=6700 \AA \lambda_{4}=5085 \AA$, $\lambda_{5}=4100 \AA$. Our model successfully predicts the harmonics as observations show. Change of free parameters alter the flux values of the harmonics but do not change their positions. One common property of the cyclotron spectra of the all the polars is that the emission source is optically thick in the lower harmonics (e.g., Wickramasinghe \& Meggitt 1982; Woelk \& Beuermann 1995). 


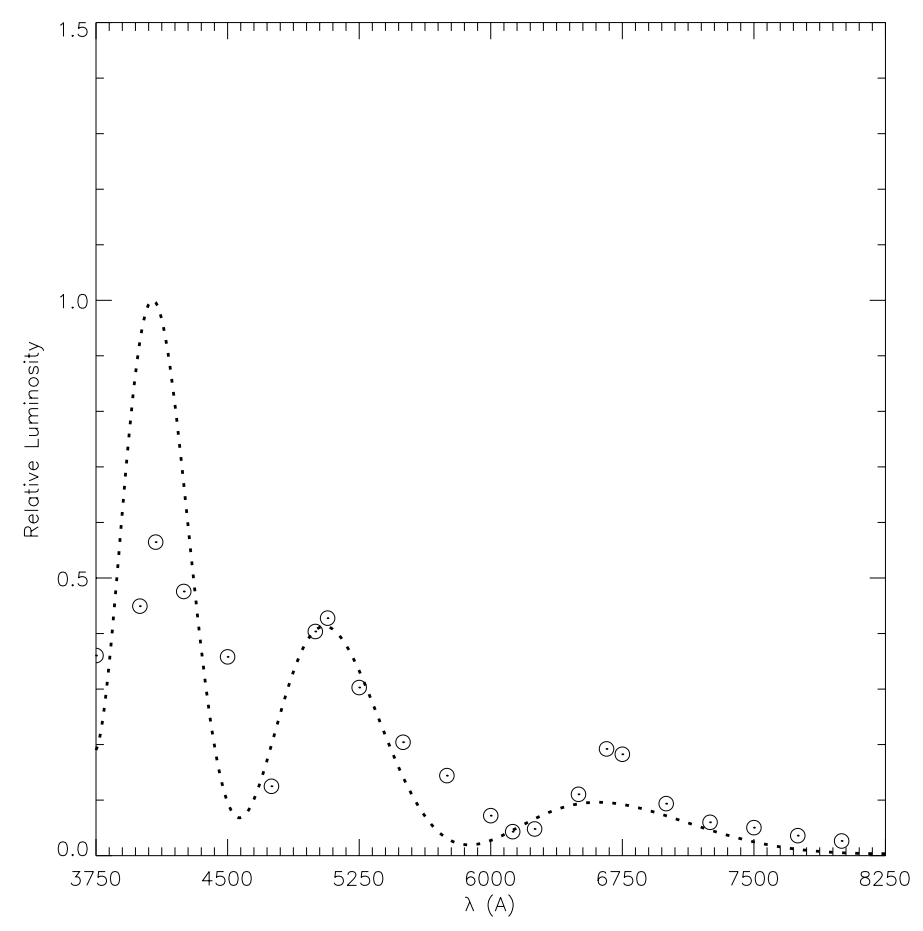

Fig. 1. The solution of Eq. (25) (dotted line) and the data (circles with dots) acquired by graphical reading from Schwope et al. (1990).

We adopted $\alpha_{\|} / \alpha_{\perp}=2.236$, which implies that in the emission region $v_{\perp} / v_{\|}=\sqrt{5}$. Physically, this assumption implies that in the region of capture by magnetic field (i.e., where free fall of streaming particles ends and the guidance of the magnetic field begins), the majority of particles lie outside the local loss-cone in such a way as to find their respective mirror points within the radiation slab. As is well-known the closer a charged particle approaches its mirror point, the greater the $v_{\perp}$ component grows at the expense of the $v_{\|}$one. By changing the value of this ratio to $v_{\perp} / v_{\|}=\sqrt{2}$ we observe that the luminosity decreases to 5.31 th of the initially adopted value (i.e., $v_{\perp} / v_{\|}=\sqrt{5}$ ) for the 5 th harmonic of UZ For. It is also implicit in Eqs. (25) and (26) that the greater the $\alpha_{\|} / \alpha_{\perp}$ ratio, the more luminous the ordinary mode. If reliable polarization measurements can be made of the pure emission then a tight constraint may be put on the $\alpha_{\|} / \alpha_{\perp}$ ratio. Because the ordinary mode is always linearly polarized, the extraordinary mode is circular polarization.

\subsection{Some general properties}

In this subsection we intend to show how the cyclotron radiation luminosity depends on the viewing angle (Fig. 2), electron number density, electron temperature, and $\alpha_{\|} / \alpha_{\perp}$ ratio, and finally on the polarization properties of the cyclotron radiation (Figs. 3a-c).

Schwope et al. (1993) and Schwope (1995) argue that, due to the advantageous geometry shown by MR Ser, our los forms a right angle with the cyclotron emission region at one face, and a half period later we seem to look down along the accretion column. In the latter case, the optical brightness is reported to reach its minimum. This is attributed to the anisotropy of the cyclotron radiation, which is confined in a narrow solid angle around the instantaneous velocity vector, which itself is more or less perpendicular to the local magnetic field.

Our Fig. 2 also shows this well-known property of radiation quite clearly; i.e., the larger the angle between the los and the magnetic field, the more luminous the source. Since the composite spectrum we observe is produced by contribution from WD, $\mathrm{RD}$, accretion streams, and the accretion column, the lesser luminosity from the cyclotron radiation may make it hard to identify the harmonics in the spectrum. Indeed Fig. 3 in Schwope's (1995) study shows this fact clearly. The upper two spectra of MR Ser shown there were taken $50 \mathrm{~min}$ apart; hence, they belong to the same low state of accretion. But the viewing angle to the cyclotron emission region changed considerably. When we look down along the field lines the optical brightness reaches its minimum, due to the anisotropy of the cyclotron radiation. It is well-known from polar diagrams of cyclotron radiation (see, e.g., Bekefi 1966) that the emission is progressively confined to a narrower cone (whose axis is perpendicular to the magnetic field) as one observes the higher harmonics. For instance, for the 5 th harmonic the apex angle of the radiation cone is about $60^{\circ}$, while the same angle for the second harmonic is about $120^{\circ}$. A similar argument comes from Ferrario et al. (2003), who attributed the change in the flux level of $1730 \AA$ feature to the viewing angle. Again, when outlining the theoretical and empirical framework among many other properties, Szkody et al. (2003) touch upon the strong dependence of cyclotron opacity on viewing angle. This property is also successfully demonstrated by our model. 


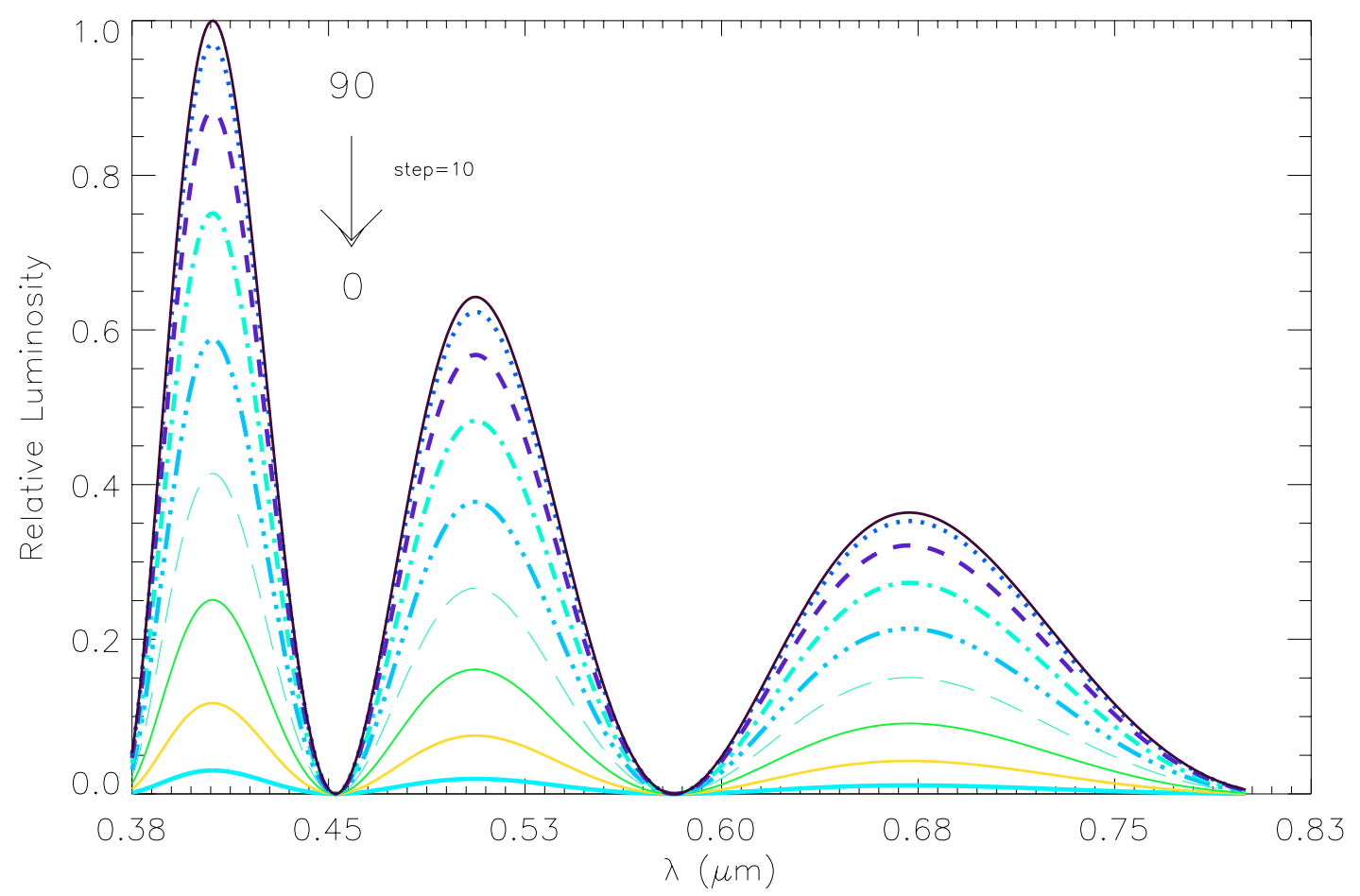

Fig. 2. Angular dependence of cyclotron radiation flux. Luminosity of the 5 th harmonic is more sensitive than those of the 4 th and 3 rd.

Figures 3a,b show the variation of luminosity with respect to $N_{\mathrm{e}}$, and $T_{\mathrm{e}}$, respectively. It is apparent from Fig. 3a that the luminosity decreases with the decrease in number density, while the reverse change occurs in the case of electron temperature; i.e. we get more luminosity from lower energy particles. As to the effect of $v_{\perp} / v_{\|}$ratio Fig. $3 \mathrm{c}$ shows an interesting result: the higher the ratio, the more luminous the source becomes. The bottom spectra, but one, is for a Maxwellian velocity distribution function. The bottom spectra is for $v_{\perp} / v_{\|}<1$, which shows that the observer whose los is more or less perpendicular to the accretion column receives more cyclotron luminosity than otherwise. Figure $3 \mathrm{c}$ also shows the effect of a smooth passage from a bi-Maxwellian to a Maxwellian distribution. This passage shows that the spectra is recovered for a Maxwellian only with lower luminosity.

A distinguishing characteristic of AM Herculis systems is strong circular polarization $(\sim 10 \%)$ observed in the optical and infrared bands. Such polarized radiation is believed to be produced by cyclotron emission arising from the accretion column.

The Stokes parameters $I, Q, U$, and $V$ are given by following:

$I=I_{+}+I_{-}$,

$Q=I_{+}\left(\frac{1-a_{\theta+}^{2}}{1+a_{\theta+}^{2}}\right)-I_{-}\left(\frac{1-a_{\theta-}^{2}}{1+a_{\theta-}^{2}}\right)$,

$U=0$,

$V=2\left(\frac{I_{+} a_{\theta+}}{1+a_{\theta+}^{2}}+\frac{I_{-} a_{\theta-}}{1+a_{\theta-}^{2}}\right)$

where $a_{\theta-}$ and $a_{\theta+}$ are the polarization coefficients for two modes (ordinary (+) and extraordinary ( - )) and $\theta$ is the angle between the direction of propagation of the electromagnetic wave and the magnetic field $B$. The circular polarization and linear polarization are given by $V / I$ and $Q / I$, respectively (see, e.g. Wu \& Chanmugam 1989).

To test our model polarization predictions, we used BL Hyi data. Figure 4 shows the result. The polarization data was adopted from Piirola et al. (1987), and circular polarization data from Wu \& Chanmugam (1989). Since our model predicts a higher linear polarization degree than observed we reduced the model values by a factor of 3.4. A similar reduction is pursued by Piirola et al. (1987) for BL Hyi. Our model circular polarization values are not reduced at all. We believe there is a good fit between our model results and observed values. We, unfortunately, cannot test our prediction with observational data for UZ For, since the recent polarization measurements on AM Her systems tend to be done with respect to the orbital phases. As we already pointed out above, we are not looking into the temporal evolution of the radiation, since we are not in a position to use those data. Last but not least, our model does not take the mode coupling and possible boundary effects at the edge of the accretion column into consideration; under some certain conditions these might affect the emergent polarization (Melrose 1978). 


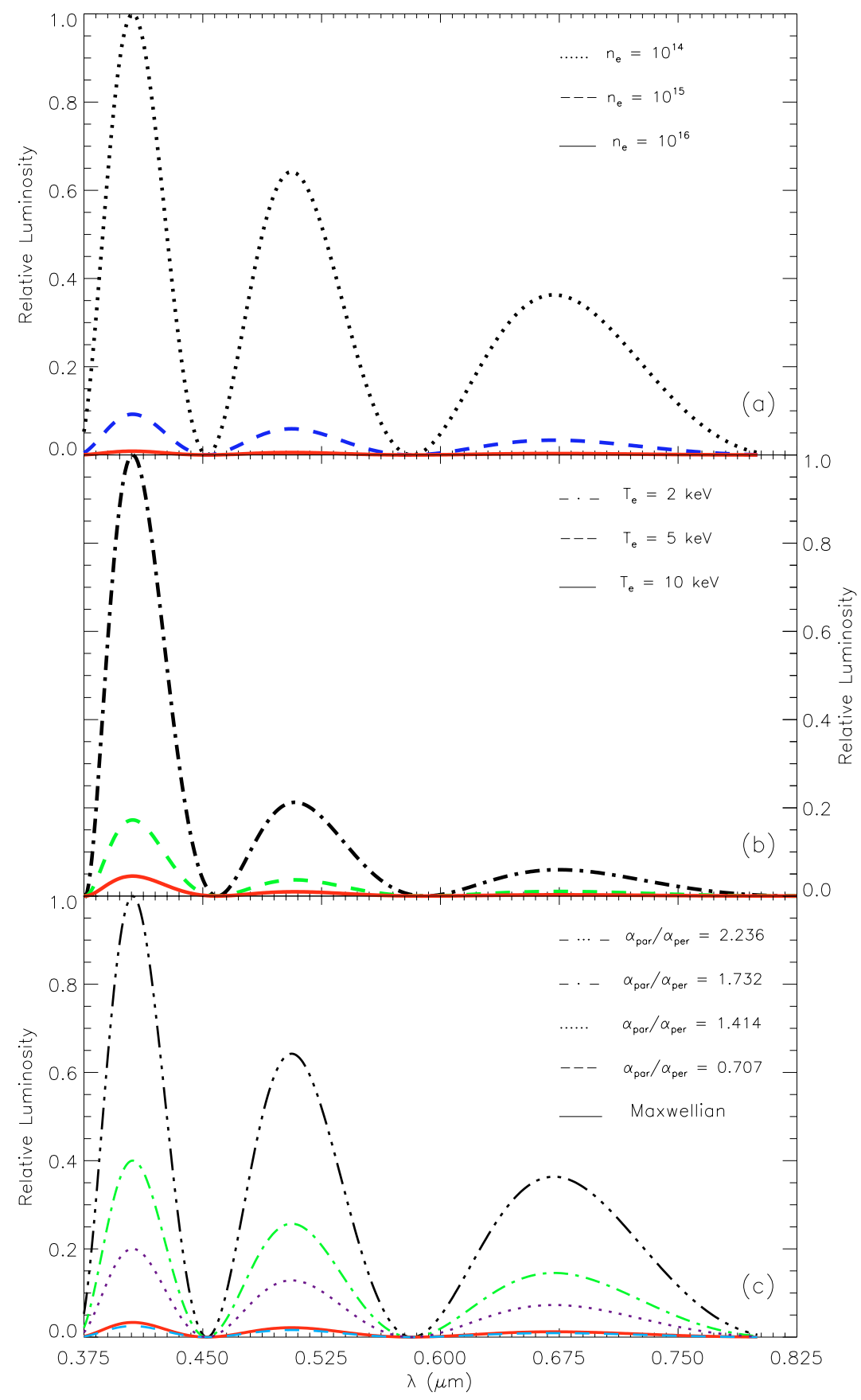

Fig. 3. a) The variation of luminosity with respect to particle number density; b) the variation of luminosity with respect to electron temperature; c) the variation of the flux level with respect to the $\alpha_{\|} / \alpha_{\perp}$ ratio. If the perpendicular (per) component of the velocity vector is higher than the parallel (par) one, the observer whose los is perpendicular to the magnetic field in the accretion column receives more luminosity; similar conditions, with a Maxwellian distribution give out less luminosity; but even lesser luminosity is received when the parallel component of the velocity vector is greater than the perpendicular one.

\section{Summary and conclusions}

We were motivated in the present study by the Schwope et al. (1990) proposal that in the UZ For case the electron velocity distribution non-Maxwellian. This is physically quite a sound suggestion when one considers this highly magnetized medium as the magnetic polar region of a magnetic CV. If accretion is controlled by the magnetic field, then the dynamical properties of accreting material are determined predominantly by the Lorentz forces. During low mass accretion times shocks cannot form and particles spiral down and go through Coulomb collisions. Near the magnetic polar regions of WD, particles seek their respective mirror points. But this is not to say that accretion will not take place. Cyclotron cooling, collisions, and streaming particles from behind will cause precursors to go through pitch angle diffusion rapidly and precipitate onto WD surface. Observations 

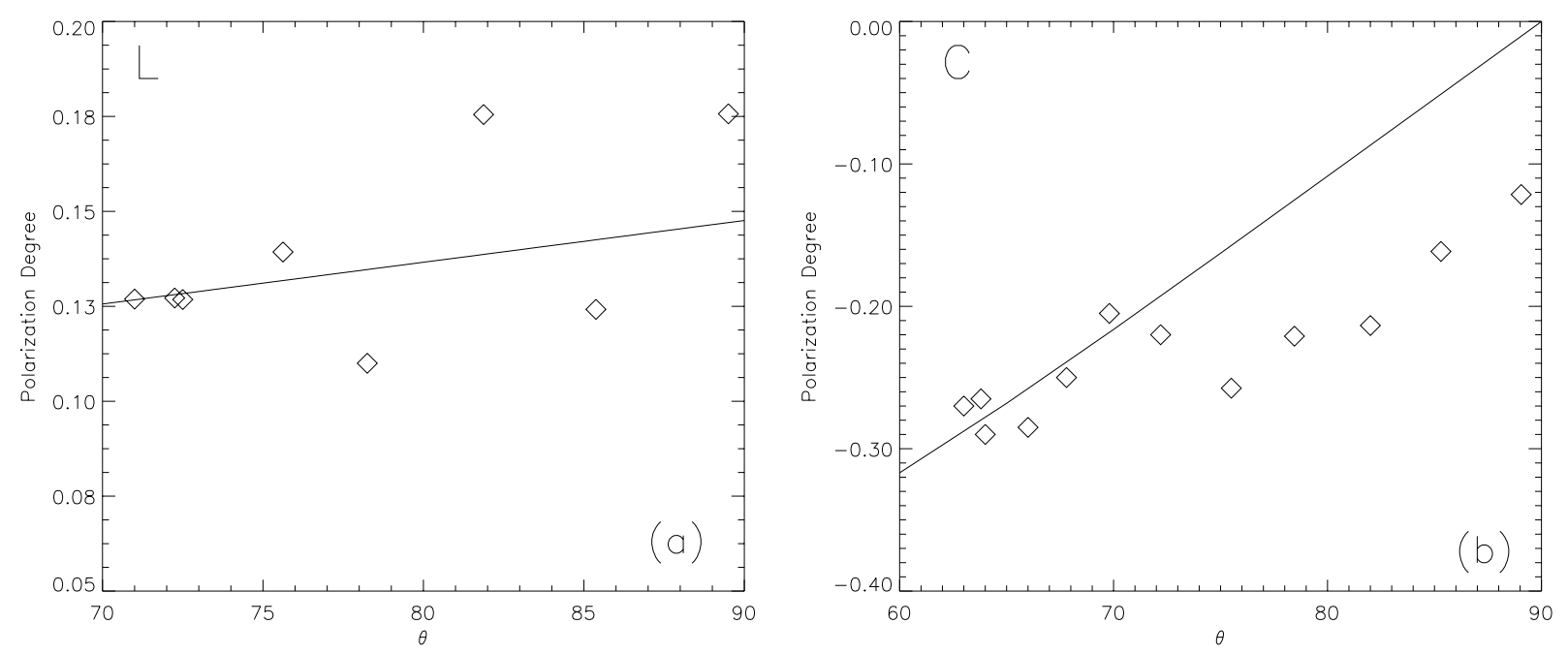

Fig. 4. Polarization comparison of model predictions with observations of BL Hyi. Diamonds are observational data and continuous lines are model predictions: a) for linear and b) for circular polarizations. We reduced the model values for linear polarization by a factor of 3.4 .

indicate that cyclotron radiation is emitted by electrons that are close to their mirror points. Angular distribution of their radiation field is such that waves propagate nearly perpendicularly to the external magnetic field. Therefore we assumed that the escaping radiation is in either extraordinary $(\mathrm{X})$ or ordinary $(\mathrm{O})$ (or both) modes. These electrons radiate in $\mathrm{X}$ and/or $\mathrm{O}$ modes in a highly dispersive medium. Dispersion relations of these modes contain the velocity distribution function and its derivatives. We thought, after having been motivated by the Schwope et al. suggestion, that the velocity distribution function of radiating electrons assume a bi-Maxwellian form with a perpendicular component of velocity much greater than the parallel one. We set $\alpha_{\perp}$ and $\alpha_{\|}$, the inverses of most probable speeds in the perpendicular and parallel directions, respectively, as free parameters. Dispersion relations then were substituted into the Green function. Once the Green function is known, one can find the radiation field and the Poynting vector, and then determine the instantaneous energy flux. The results of the solution are given in Fig. 1, and contains a series of free parameter ranges, $10^{14} \leq n_{\mathrm{e}} \leq 10^{16} \mathrm{~cm}^{-3}$ and $1 \mathrm{keV} \leq k T \leq 10 \mathrm{keV}$. The distribution of flux with respect to wavelength changes with the change of one parameter. But this change can easily be compensated for by the change of another parameter. Therefore we did not show all these changes in the figures. Instead we aimed at reproducing the observed spectra by suitable choices of free parameters. We tested our model on UZ For, whose observed spectrum is quite successfully reproduced.

The strong points of the model are: (i) all the harmonics that are observed are reproduced at their central wavelengths with high precision; (ii) line widths $(\Delta \lambda \sim 1000 \AA$ ) are also successfully reproduced; (iii) cyclotron harmonics of UZ For display a steep rise on the blue wing and a rather mild decrease on the red. This property is successfully reproduced for UZ For case (see especially for the 4th harmonic); (iv) our result on the dominance of the O-mode for the the cyclotron emission is completely the same as the one in Chanmugam \& Dulk (1981). As for the weak point of the model, the plasma column is assumed to be isothermal, homogeneous, and permeated by a uniform magnetic field with no spatial variation. These assumptions may be regarded as physically unrealistic. Nevertheless, as a first approximation, the model seems to be consistent with observations.

Acknowledgements. The authors are grateful to P. Szkody, A. D. Schwope, K. Wu, A. Gimenez, P. Harmanec, and H. Livaniou-Rovithis for valuable discussions, and to D. Reimers and H.-J. Hagen for sharing their data with us. This work was supported by Ege University Research Fund as a part of the Ph.D. study of BK (Project No. 2002/FEN/002) and by TÜBİTAK. Finally, we express our thanks to the anonymous referee for her/his helpful, constructive criticism that helped to improve this paper.

\section{References}

Bailey, J., Watts, D. J., Sherrington, M. R., et al. 1985, MNRAS, 215, 179

Bailey, J., Ferrario, L., Tuohy, I. R., Wickramasinghe, D. T., \& Hough, J. H. 1987, IAU Circ., No. 4517

Barrett, P. E., \& Chanmugam, G. 1984, ApJ, 278, 298

Barrett, P. E., \& Chanmugam, G. 1985, ApJ, 298, 743

Bekefi, G. 1966, Radiation Processes in Plasmas (NY: John Wiley \& Sons Inc.)

Beuermann, K., Thomas, H.-C., \& Schwope, A. 1987, IAU Circ., No. 4517

Boyd, T. J. M., \& Sanderson, J. J. 1969, Plasma Dynamics (London: NELSON)

Burwitz, V., Reinsch, K., Beuermann, K., \& Thomas, H.-C. 1997, A\&A, 327, 183

Chanmugam, G., \& Dulk, G. A. 1981, ApJ, 244, 569

Chanmugam, G., \& Langer, S. H. 1991, ApJ, 368, 580

Ferrario, L., Bailey, J., \& Wickramasinghe, D. 1996, MNRAS, 282, 218 
Ferrario, L., Wickramasinghe, D. T., Schmidt, G. 2003, MNRAS, 338, 340

Giommi, P., Angelini, L., Osborne, J., et al. 1987, IAU Circ., No. 4486

Jackson, J. D. 1975, Classical Electrodynamics (NY: John Wiley and Sons Inc.), 223

Kuijper, J., \& Pringle, J. E. 1982, A\&A, 114, L4

Lamb, D. Q., \& Masters, J. E. 1979, ApJ, 234, 117

Meggitt, S. M. A., \& Wickramasinghe, D. T. 1982, MNRAS, 198, 71

Melrose, D. B. 1978, Proc. Astr. Soc. Aust., 3, 229

Osborne, J. P., Giommi, P., Angelini, L., Tagliaferri, G., \& Stella, L. 1988, ApJ, 328, 45

Pacholczyk, A. G. 1970, Radio Astrophysics (San Francisco: W. H. Freeman and Company)

Piirola, V., Coyne, G. V., \& Reiz, A. 1987, A\&A, 185, 189

Ramaty, R. 1969, ApJ, 158, 753

Reimers, D., Hagen, H.-J., \& Hopp, U. 1999, A\&A, 343, 157

Rousseau, Th., Fischer, A., Beuermann, K., \& Woelk, U. 1996, A\&A, 310, 526

Schmidt, G. 1979, Physics of High Temperature Plasmas, 2nd ed. (NY: AP)

Schmidt, G. D., Stockman, H. S., \& Grandi, S. A. 1983, ApJ, 271, 735

Schwope, A. D. 1995, Rev. Mod. Astron., 8, 125

Schwope, A. D., Beuermann, K., \& Thomas, H.-C. 1990, A\&A, 230, 120

Schwope, A. D., Beuermann, K., Jordan, S., \& Thomas, H.-C. 1993, A\&A, 278, 487

Schwope, A. D., Beuermann, K., \& Jordan, S. 1995, A\&A, 301, 447

Schwope, A. D., Brunner, H., Hambaryan, V., \& Schwarz, R. 2002, ASP Conf. Ser., 261, 102

Szkody, P., Anderson, S. F., Schmidt, G., et al. 2003, ApJ, 583, 902

Visvanathan, N., \& Wickramasinghe, D. T. 1979, Nature, 281, 47

Vocks, C., \& Marsch, E. 2002, ApJ, 568, 1030

Wickramasinghe, D. T., \& Meggitt, S. M. A. 1982, MNRAS, 198, 975

Wickramasinghe, D. T., \& Ferrario, L. 1995, ASP Conf. Ser., 85, 43

Woelk, U., \& Beuermann, K. 1992, A\&A, 256, 498

Woelk, U., \& Beuermann, K. 1993, A\&A, 280, 169

Woelk, U., \& Beuermann, K. 1995, ASP Conf. Ser., 85, 215

Woelk, U., \& Beuermann, K. 1996, A\&A, 306, 232

Wu, C. S. 1985, Space Sci. Rev., 41, 215

Wu, K., \& Chanmugam, G. 1989, ApJ, 344, 889

Wu, K., \& Wickramasinghe, D. T. 1992, ASP Conf. Ser., 29, 203 